\title{
UNSTEADY FLOW THROUGH A POROUS MEDIUM DUE TO NON- COAXIAL ROTATIONS OF A POROUS DISK AND A FLUID AT INFINITY SUBJECTED TO A PERIODIC SUCTION
}

\author{
M. GURIA \\ Department of Mathematics, Ghatal Rabindra Satabarsiki Mahavidyalaya \\ Ghatal, Paschim Medinipur, West Bengal, INDIA \\ E-mail: mrinmoy9832@yahoo.com
}

\begin{abstract}
The unsteady flow of a viscous incompressible fluid due to non-coaxial rotations of a porous disk and a fluid at infinity subjected to a periodic suction through a porous medium has been studied. The velocity field, shear stresses are obtained in closed form. The variations of primary and secondary velocities for different values of non dimensional parameters are depicted in figures.
\end{abstract}

Key words: periodic suction, non-coaxial, eccentric, oscillating, rotating.

\section{Introduction}

The flow of a viscous incompressible fluid due to non-coaxial rotations of a disk and a fluid at infinity has been studied by a number of researchers. An exact solution of this type of problem was obtained by Berker [1]. Coirier [2] studied the flow due to a disk and a fluid at infinity which are rotating noncoaxially at a slightly different angular velocity. The non-Newtonian flow due to a disk and a fluid at infinity which are rotating non-coaxially at a slightly different angular velocity was studied by Erdogan [3]. An exact solution of the three dimensional Navier-Stokes equations for the flow due to non-coaxial rotations of a porous disk and a fluid at infinity was studied by Erdogan $[4,5]$. The unsteady flow due to non-coaxial rotations of a disk, oscillating in its own plane and a fluid at infinity was studied by Kasiviswanathan and Rao [6]. The flow due to non-coaxial rotations of an oscillatory porous disk and a fluid at infinity about an axis passing through a fixed point parallel to the axis of rotation of the disk was investigated by Hayet et al. [7]. The flow due to non-coaxial rotations of an oscillating porous disk and a fluid at infinity which rotate about an axis passing through a fixed point parallel to the axis of rotation of the disk was studied by Guria et al. [8]. Guria et al. [9] investigated the Hall effects on an unsteady flow of a viscous fluid due to non-coaxial rotations of a porous disk and a fluid at infinity. Ghosh et al. [10] studied the flow due to non coaxial rotations of a porous disk subjected to a periodic suction and a fluid at infinity. Guria et al. [11] studied the flow due to non-coaxial rotations of a porous disk and a fluid at infinity through a porous medium. Hayat et al. [12] also studied the unsteady MHD flow due to non-coaxial rotations of a porous disk and a fluid at infinity. The effects of Hall currents on the unsteady MHD flow due to non-coaxial rotations of a porous disk and a fluid at infinity were studied by Hayat et al. [13]. Ghara et al. [14] also investigated the Hall effects on the viscous incompressible fluid due to non-coaxial rotations of an oscillating porous disk and a fluid at infinity.

In the present paper, the effect of permeability on an unsteady flow due to non-coaxial rotations of a porous disk subjected to a periodic suction or blowing and a fluid at infinity is studied. 


\section{Formulation of the problem and its solution}

Consider an unsteady flow of a viscous incompressible fluid embedded in a porous medium occupying the space $z>0$ and is bounded by an infinite porous disk at $z=0$. The axes of rotation of both the disk and that of the fluid at infinity are in the plane $x=0$. The distance between the axes of rotation is $l$. Initially, at $t=0$, the disk and the fluid at infinity rotate about the $z^{\prime}-$ axis with the same uniform angular velocity $\Omega$. At time $t>0$, the disk suddenly starts to rotate about the $z$ - axis with uniform angular velocity while the fluid at infinity continues to rotate about the $z^{\prime}-$ axis with the same angular velocity as that of the disk.

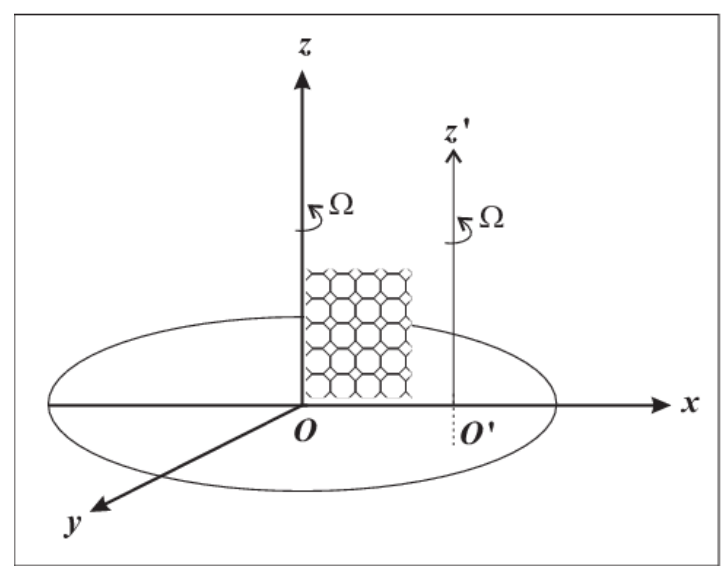

Fig.1. Geometry of the problem.

The boundary conditions of the problem are

$$
\begin{aligned}
& u=-\Omega(y-l), \quad v=\Omega x, \quad w=-V(t) \quad \text { at } \quad t=0, \quad z>0, \\
& u=-\Omega y, \quad v=\Omega x, \quad w=-V(t) \quad \text { at } \quad z=0, \quad t>0, \\
& u=-\Omega(y-l), \quad v=\Omega x \quad \text { as } \quad z \rightarrow \infty \quad \text { for all } t .
\end{aligned}
$$

The Navier Stokes eqations are, respectively

$$
\begin{aligned}
& \frac{\partial u}{\partial t}+u \frac{\partial u}{\partial x}+v \frac{\partial u}{\partial y}+w \frac{\partial u}{\partial z}=-\frac{1}{\rho} \frac{\partial p}{\partial x}+v\left(\frac{\partial^{2} u}{\partial x^{2}}+\frac{\partial^{2} u}{\partial y^{2}}+\frac{\partial^{2} u}{\partial z^{2}}\right)-\frac{\mu}{\rho k} u \\
& \frac{\partial v}{\partial t}+u \frac{\partial v}{\partial x}+v \frac{\partial v}{\partial y}+w \frac{\partial v}{\partial z}=-\frac{1}{\rho} \frac{\partial p}{\partial y}+v\left(\frac{\partial^{2} v}{\partial x^{2}}+\frac{\partial^{2} v}{\partial y^{2}}+\frac{\partial^{2} v}{\partial z^{2}}\right)-\frac{\mu}{\rho k} v \\
& \frac{\partial w}{\partial t}+u \frac{\partial w}{\partial x}+v \frac{\partial w}{\partial y}+w \frac{\partial w}{\partial z}=-\frac{1}{\rho} \frac{\partial p}{\partial z}+v\left(\frac{\partial^{2} w}{\partial x^{2}}+\frac{\partial^{2} w}{\partial y^{2}}+\frac{\partial^{2} w}{\partial z^{2}}\right)-\frac{\mu}{\rho k} w
\end{aligned}
$$


where $(u, v, w)$ are the velocity components along the coordinate axes, $\rho$ is the fluid density, $v$ is the kinematic coefficient of viscosity. The perodic suction velocity distibution of the form

$$
V(t)=-w_{0}\left[1+\varepsilon A e^{i \sigma t}\right]
$$

where $w_{0}$ is a positive constant, $\varepsilon>0$ is very small and $\mathrm{A}$ is a real positive constant such that $\varepsilon A \leq 1$.

We assume the velocity components of the form

$$
u=-\Omega y+f(z, t), \quad v=\Omega x+g(z, t), \quad w=-V(t) .
$$

Using Eq.(2.6), the equations of motion become

$$
\begin{aligned}
& \frac{\partial f}{\partial t}-V(t) \frac{\partial f}{\partial z}-\Omega g=-\frac{1}{\rho} \frac{\partial p}{\partial x}+\Omega^{2} x+\frac{\mu \Omega}{\rho k} y+v \frac{\partial^{2} f}{\partial z^{2}}-\frac{\mu}{\rho k} f, \\
& \frac{\partial g}{\partial t}-V(t) \frac{\partial g}{\partial z}+\Omega f=-\frac{1}{\rho} \frac{\partial p}{\partial y}+\Omega^{2} y-\frac{\mu \Omega}{\rho k} x+v \frac{\partial^{2} f}{\partial z^{2}}-\frac{\mu}{\rho k} f, \\
& \frac{\partial V(t)}{\partial t}=\frac{1}{\rho} \frac{\partial p}{\partial z}-\frac{\mu}{\rho k} V(t) .
\end{aligned}
$$

Differentiating Eqs (2.7) and (2.8), we get

$$
\begin{aligned}
& \frac{\partial^{2} f}{\partial z \partial t}-V(t) \frac{\partial^{2} f}{\partial z^{2}}-\Omega \frac{\partial g}{\partial z}=v \frac{\partial^{3} f}{\partial z^{3}}-\frac{\mu}{\rho k} \frac{\partial f}{\partial z} \\
& \frac{\partial^{2} g}{\partial z \partial t}-V(t) \frac{\partial^{2} g}{\partial z^{2}}-\Omega \frac{\partial f}{\partial z}=v \frac{\partial^{3} g}{\partial z^{3}}-\frac{\mu}{\rho k} \frac{\partial g}{\partial z}
\end{aligned}
$$

Combining Eqs (2.10) and (2.11), we get

$$
v \frac{\partial^{3} F}{\partial z^{3}}+V(t) \frac{\partial^{2} F}{\partial z^{2}}-\frac{\partial^{2} F}{\partial z \partial t}-\left(i \Omega+\frac{\mu}{\rho k}\right) \frac{\partial F}{\partial z}=0
$$

where

$$
F=f+i g, \quad i=\sqrt{-1} .
$$

The corresponding boundary conditions become

$$
\begin{aligned}
& F=\Omega l, \quad z \geq 0, \\
& F(0, t)=0, \quad \text { for } \quad t>0
\end{aligned}
$$




$$
F(\infty, t)=\Omega l, \quad \text { for } \quad t>0 .
$$

We assume

$$
F(z, t)=F_{0}(z, t)+\varepsilon F_{l}(z, t) e^{i \sigma t}
$$

Substituitng Eq.(2.16) in Eq.(2.12) and comparing the harmonics terms and neglecting the coefficients of $\varepsilon^{2}$, we get

$$
\begin{aligned}
& \vee \frac{d^{3} F_{0}}{d z^{3}}+w_{0} \frac{d^{2} F_{0}}{d z^{2}}-\left(i \Omega+\frac{\mu}{\rho k}\right) \frac{d F_{0}}{d z}=0, \\
& \vee \frac{d^{3} F_{1}}{d z^{3}}+w_{0} \frac{d^{2} F_{1}}{\partial z^{2}}-\left(i \Omega+i \sigma+\frac{\mu}{\rho k}\right) \frac{d F_{1}}{d z}=-w_{0} A \frac{d^{2} F_{0}}{d z^{2}} .
\end{aligned}
$$

Introducing the non-dimensional variables

$$
\eta=\sqrt{\frac{\Omega}{2 v}} z, \quad S=\frac{w_{0}}{2 \sqrt{\Omega v}}, \quad n=\sqrt{1+\frac{\sigma}{\Omega}}, \quad K=\frac{k \Omega}{v},
$$

Eqs (2.17) and (2.18) become

$$
\begin{aligned}
& \frac{d^{3} F_{0}}{d \eta^{3}}+2 \sqrt{2} S \frac{d^{2} F_{0}}{d \eta^{2}}-2\left(\frac{1}{K}+i\right) \frac{d F_{0}}{d \eta}=0 \\
& \frac{d^{3} F_{1}}{d z^{3}}+2 \sqrt{2} S \frac{d^{2} F_{1}}{d \eta^{2}}-2\left(\frac{1}{K}+i \eta^{2}\right) \frac{d F_{1}}{d \eta}=-2 \sqrt{2} S A \frac{d^{2} F_{0}}{d \eta^{2}} .
\end{aligned}
$$

The boundary conditions (2.14) and (2.15) become

$$
F_{0}(0)=0, \quad F_{0}(\infty)=\Omega l, \quad F_{l}(0)=0, \quad F_{l}(\infty)=0 .
$$

Solving the Eqs (2.20) and (2.21) subject to the boundary conditions (2.22) and using Eq.(2.16), we get

$$
\begin{aligned}
& \frac{f}{\Omega l}=\left(1-e^{-\alpha_{0} \eta} \cos \beta_{0} \eta\right)+\frac{\varepsilon \sqrt{2} S A}{\left(n^{2}-1\right)}\left[\alpha_{0}\left\{e^{-\alpha_{I} \eta} \sin \left(\beta_{I} \eta-\sigma t\right)-e^{-\alpha_{0} \eta} \sin \left(\beta_{0} \eta-\sigma t\right)\right\}+\right. \\
& \left.+\beta_{0}\left\{e^{-\alpha_{0} \eta} \cos \left(\beta_{0} \eta-\sigma t\right)-e^{-\alpha_{I} \eta} \cos \left(\beta_{I} \eta-\sigma t\right)\right\}\right],
\end{aligned}
$$




$$
\begin{aligned}
& \frac{g}{\Omega l}=e^{-\alpha_{0} \eta} \sin \beta_{0} \eta+\frac{\varepsilon \sqrt{2} S A}{\left(n^{2}-l\right)}\left[\alpha_{0}\left\{e^{-\alpha_{I} \eta} \cos \left(\beta_{I} \eta-\sigma t\right)-e^{-\alpha_{0} \eta} \cos \left(\beta_{0} \eta-\sigma t\right)\right\}+\right. \\
& \left.-\beta_{0}\left\{e^{-\alpha_{0} \eta} \sin \left(\beta_{0} \eta-\sigma t\right)-e^{-\alpha_{I} \eta} \sin \left(\beta_{I} \eta-\sigma t\right)\right\}\right]
\end{aligned}
$$

where

$$
\begin{aligned}
& \alpha_{0}=\sqrt{2} S+\gamma_{0}, \quad \alpha_{1}=\sqrt{2} S+\gamma_{1}, \\
& \gamma_{0}=\left\{\left(S^{2}+\frac{1}{K}\right)+\sqrt{\left(S^{2}+\frac{1}{K}\right)^{2}+1}\right\}^{1 / 2}, \\
& \beta_{0}=\left\{-\left(S^{2}+\frac{1}{K}\right)+\sqrt{\left(S^{2}+\frac{1}{K}\right)^{2}+1}\right\}^{1 / 2}, \\
& \gamma_{1}=\left\{\left(S^{2}+\frac{1}{K}\right)+\sqrt{\left(S^{2}+\frac{1}{K}\right)^{2}+n^{4}}\right\}^{1 / 2}, \\
& \beta_{1}=\left\{-\left(S^{2}+\frac{1}{K}\right)+\sqrt{\left(S^{2}+\frac{1}{K}\right)^{2}+n^{4}}\right\}^{1 / 2} .
\end{aligned}
$$

The flow very near the porous disk is

$$
\begin{aligned}
& \frac{f}{\Omega l}=\alpha_{0} \eta+\frac{\varepsilon \sqrt{2} S A}{\left(n^{2}-1\right)}\left[\alpha_{0}\left\{\left(\beta_{1}-\beta_{0}\right) \eta \cos \sigma t+\left(\alpha_{1}-\alpha_{0}\right) \eta \sin \sigma t\right\}+\right. \\
& \left.+\beta_{0}\left\{\left(\alpha_{1}-\alpha_{0}\right) \eta \cos \sigma t-\left(\beta_{1}-\beta_{0}\right) \eta \sin \sigma t\right\}\right], \\
& \frac{g}{\Omega l}=\beta_{0} \eta+\frac{\varepsilon \sqrt{2} S A}{\left(n^{2}-1\right)}\left[\alpha_{0}\left\{\left(\beta_{1}-\beta_{0}\right) \eta \sin \sigma t-\left(\alpha_{1}-\alpha_{0}\right) \eta \cos \sigma t\right\}+\right. \\
& \left.+\beta_{0}\left\{\left(\alpha_{1}-\alpha_{0}\right) \eta \sin \sigma t+\left(\beta_{1}-\beta_{0}\right) \eta \cos \sigma t\right\}\right] .
\end{aligned}
$$

The inclination of the fluid velocity vector with the $y$ axis near $z=0$ becomes $\theta=\tan ^{-1}(C / D)$, where

$$
\begin{aligned}
& C=\beta_{0}\left(n^{2}-1\right)+\varepsilon \sqrt{2} S A\left[\alpha_{0}\left\{\left(\beta_{1}-\beta_{0}\right) \sin \sigma t-\left(\alpha_{1}-\alpha_{0}\right) \cos \sigma t\right\}+\right. \\
& +\beta_{0}\left\{\left(\beta_{1}-\beta_{0}\right) \cos \sigma t+\left(\alpha_{1}-\alpha_{0}\right) \sin \sigma t\right\}
\end{aligned}
$$




$$
\begin{aligned}
& D=\alpha_{0}\left(n^{2}-1\right)+\varepsilon \sqrt{2} S A\left[\alpha_{0}\left(\beta_{1}-\beta_{0}\right) \cos \sigma t+\left(\alpha_{1}-\alpha_{0}\right) \sin \sigma t+\right. \\
& \left.+\beta_{0}\left\{\left(\alpha_{1}-\alpha_{0}\right) \cos \sigma t-\left(\beta_{1}-\beta_{0}\right) \sin \sigma t\right\}\right] .
\end{aligned}
$$

Case I: When $S \neq 0, A=0, \theta=\tan ^{-1}\left(\beta_{0} / \alpha_{0}\right)<45^{\circ}$.

Case II: If $\sigma t=\pi / 2, S \neq 0, A \neq 0$, the inclination of the fluid velocity to the $y$ axis near the $z$ axis will be

$$
\theta=\tan ^{-1}\left[\frac{\beta_{0}\left(n^{2}-1\right)+\varepsilon \sqrt{2} S A\left\{\alpha_{0}\left(\beta_{1}-\beta_{0}\right)+\beta_{0}\left(\alpha_{1}-\alpha_{0}\right)\right\}}{\alpha_{0}\left(n^{2}-1\right)+\varepsilon \sqrt{2} S A\left\{\alpha_{0}\left(\alpha_{1}-\alpha_{0}\right)-\beta_{0}\left(\beta_{1}-\beta_{0}\right)\right\}}\right]
$$

\section{Results and discussion}

We have presented the non-dimensional primary velocity $f / \Omega l$ and the secondary velocity $g / \Omega l$ against $\eta$ for several values of the permeability parameter $K$, the suction or blowing parameter $S$, the magnitude of fluctuation of suction velocity $A$, and $\sigma t$. Figure 2 represents the variations of $f / \Omega l$ and the secondary velocity $g / \Omega l$ for several values of the permeability parameter $K$. It is observed that the primary velocity decreases but the secondary velocity increases with an increase in the permeability parameter. Figure 3 shows the variations of the primary velocity for several values of $S$. It is seen that suction creates thickening of the boundary layer. The crossing of the graphs shown in the figure is due to the presence of suction because suction evokes thickening of the boundary layer near the disk and thinning of the boundary layer away from the disk. From Fig.4 it is observed that both primary and secondary velocities increase with an increase in $A$. In Fig. 5 the the variations of primary and secondary velocities for different values of $\sigma t$ are plotted. It is observed that primary velocity decreases with an increase in $\sigma t$ and a reverse effect is observed for secondary velocity. Using Eqs (2.26) and (2.27), we get the non-dimensional shear stress components $\tau_{x}$ and $\tau_{y}$ at the disk when $\eta=0$ as

$$
\begin{aligned}
& \left(\tau_{x}\right)_{\eta=0}=\frac{\partial}{\partial \eta}\left(\frac{f}{\Omega l}\right)=\alpha_{0}-\frac{\varepsilon \sqrt{2} S A}{\left(n^{2}-1\right)}[P \cos \sigma t-Q \sin \sigma t], \\
& \left(\tau_{y}\right)_{\eta=0}=\frac{\partial}{\partial \eta}\left(\frac{g}{\Omega l}\right)=\beta_{0}-\frac{\varepsilon \sqrt{2} S A}{\left(n^{2}-1\right)}[Q \cos \sigma t+P \sin \sigma t]
\end{aligned}
$$

where

$$
P=\alpha_{0}\left(\beta_{0}-\beta_{1}\right)+\beta_{0}\left(\alpha_{0}-\alpha_{1}\right) \text { and } Q=\beta_{0}\left(\beta-\beta_{0}\right)-\alpha_{0}\left(\alpha_{0}-\alpha_{1}\right) \text {. }
$$

The non-dimensional shear stresses $\tau_{x}$ and $\tau_{y}$ due to the primary and secondary flows at the disk when $\eta=0$ are entered in Tab. 1 for different values of $S$ and $K$ with $\sigma t=45^{\circ}, A=2, n^{2}=1.5, \varepsilon=0.1$. 


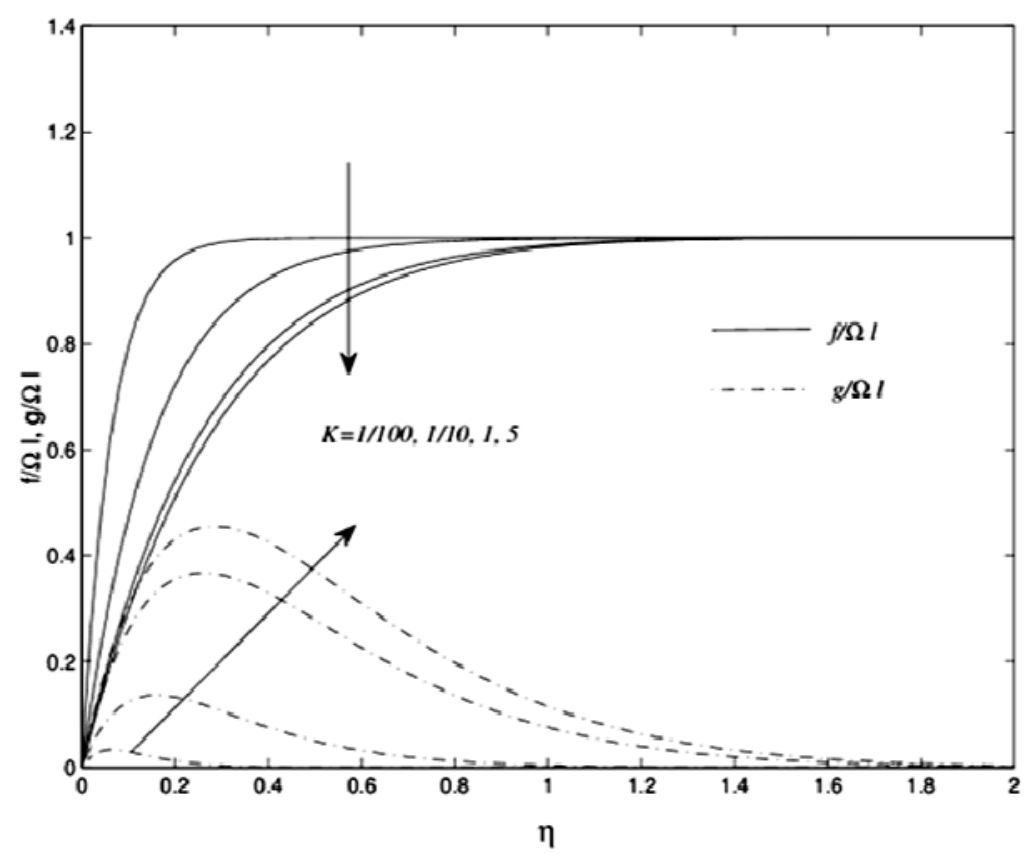

Fig.2. Variation of $f / \Omega l$ and $g / \Omega l$ for $S=1, A=2, \sigma t=45^{\circ}, \varepsilon=0.1, n^{2}=1.5$.

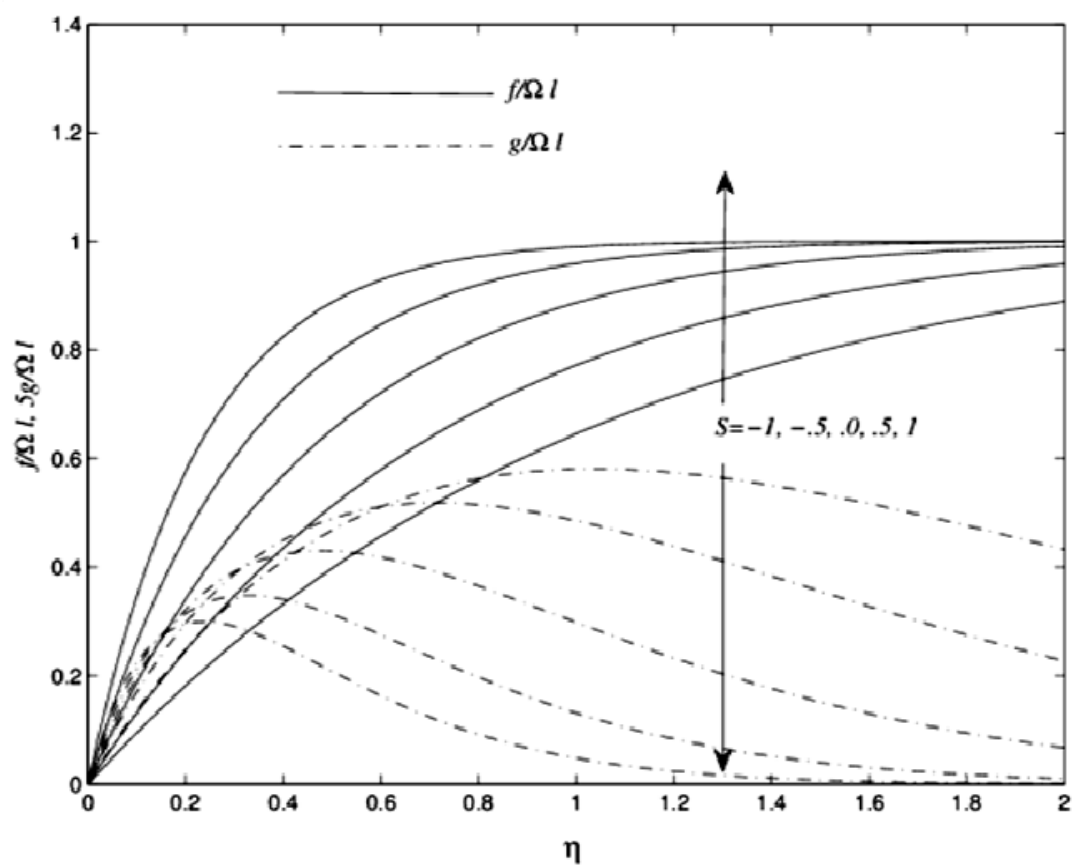

Fig.3. Variation of $f / \Omega l$ and $g / \Omega l$ for $K=0.5, A=2, \sigma t=45^{\circ}, \varepsilon=0.1, n^{2}=1.5$. 


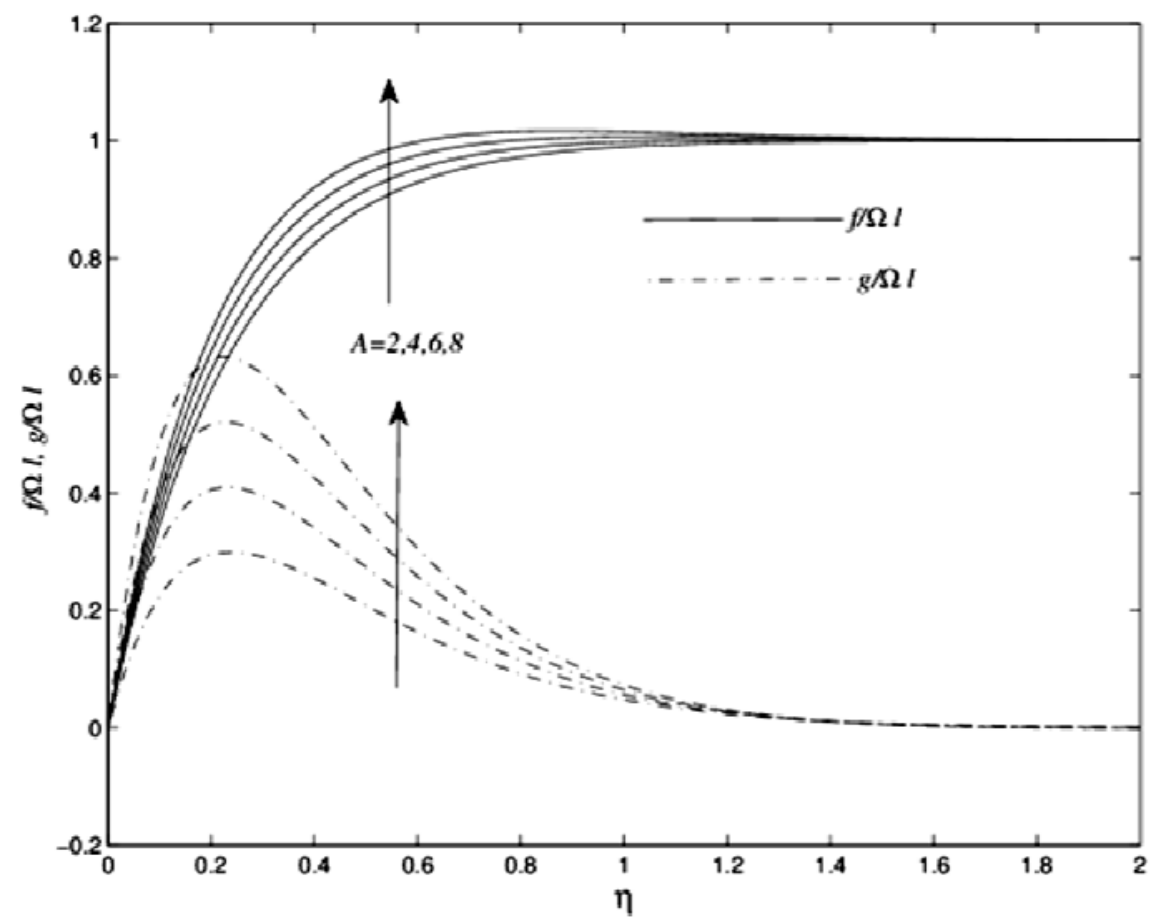

Fig.4. Variation of $f / \Omega l$ and $g / \Omega l$ for $S=1, K=0.5, \sigma t=45^{\circ}, \varepsilon=0.1, n^{2}=1.5$.

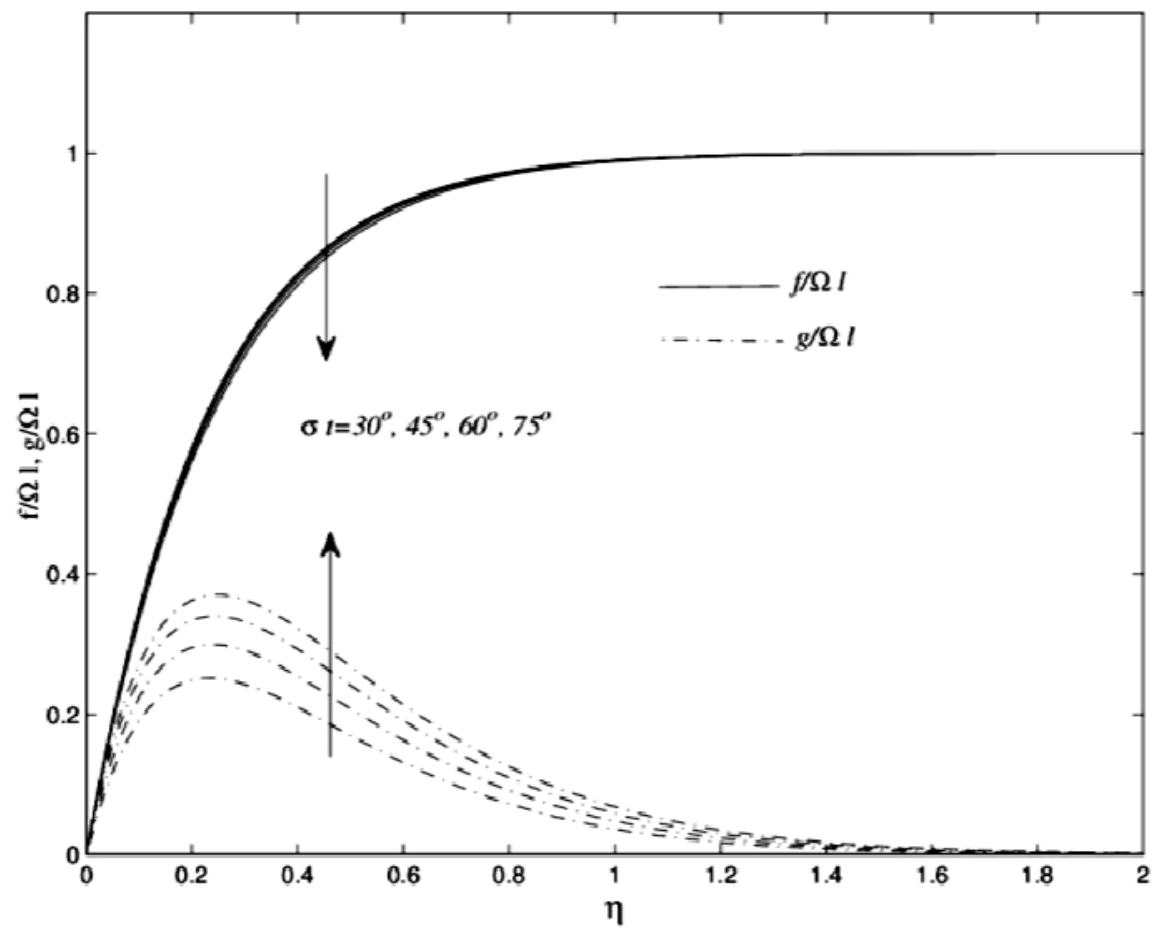

Fig.5. Variation of $f / \Omega l$ and $g / \Omega l$ for $S=1, A=2, K=0.5, \varepsilon=0.1, n^{2}=1.5$. 
Table 1. Shear stresses due to the primary and secondary flows for $A=2, \sigma t=45^{\circ}$.

\begin{tabular}{|c|c|c|c|c|c|c|c|c|}
\hline & \multicolumn{5}{|c|}{$\tau_{x}$} & \multicolumn{4}{c|}{$\tau_{y}$} \\
\hline$S \backslash K$ & $1 / 100$ & $1 / 10$ & 1 & 5 & $1 / 100$ & $1 / 10$ & 1 & 5 \\
\hline-1.0 & 12.61 & 3.12 & 0.51 & 0.12 & -0.10 & 0.09 & 0.47 & 0.62 \\
\hline-0.5 & 13.35 & 3.73 & 0.87 & 0.42 & -0.02 & 0.14 & 0.58 & 0.80 \\
\hline 0 & 14.14 & 4.47 & 1.55 & 1.10 & 0.07 & 0.22 & 0.64 & 0.90 \\
\hline 0.5 & 14.97 & 5.36 & 2.60 & 2.18 & 0.17 & 0.32 & 0.64 & 0.82 \\
\hline 1.0 & 15.84 & 6.39 & 3.92 & 3.59 & 0.28 & 0.44 & 0.67 & 0.74 \\
\hline
\end{tabular}

Table 2. Shear stresses due to the primary and secondary flows for $K=0.5, A=2$.

\begin{tabular}{|c|c|c|c|c|c|c|}
\hline & \multicolumn{3}{|c|}{$\tau_{x}$} & \multicolumn{3}{c|}{$\tau_{y}$} \\
\hline$S \backslash \sigma t$ & $30^{\circ}$ & $45^{\circ}$ & $60^{\circ}$ & $30^{\circ}$ & $45^{\circ}$ & $60^{\circ}$ \\
\hline-1.0 & 0.92 & 0.93 & 0.94 & 0.39 & 0.36 & 0.32 \\
\hline-0.5 & 1.35 & 1.36 & 1.37 & 0.45 & 0.42 & 0.40 \\
\hline 0 & 2.05 & 2.05 & 2.05 & 0.48 & 0.48 & 0.48 \\
\hline 0.5 & 3.07 & 3.05 & 3.03 & 0.48 & 0.53 & 0.58 \\
\hline 1.0 & 4.33 & 4.29 & 4.22 & 0.50 & 0.61 & 0.71 \\
\hline
\end{tabular}

Table 3. Shear stresses due to the primary and secondary flows for $\sigma t=45^{\circ}, S=1.0$.

\begin{tabular}{|c|c|c|c|c|c|c|c|c|}
\hline & \multicolumn{5}{|c|}{$\tau_{x}$} & \multicolumn{4}{c|}{$\tau_{y}$} \\
\hline$A \backslash K$ & $1 / 100$ & $1 / 10$ & 1 & 5 & $1 / 100$ & $1 / 10$ & 1 & 5 \\
\hline 2.0 & 15.84 & 6.39 & 3.92 & 3.59 & 0.28 & 0.44 & 0.67 & 0.74 \\
\hline 3.0 & 15.96 & 6.53 & 4.14 & 3.85 & 0.39 & 0.56 & 0.76 & 0.81 \\
\hline 4.0 & 16.07 & 6.67 & 4.37 & 4.11 & 0.50 & 0.68 & 0.86 & 0.88 \\
\hline 5.0 & 16.18 & 6.81 & 4.59 & 4.37 & 0.61 & 0.80 & 0.95 & 0.95 \\
\hline
\end{tabular}

It is observed that the shear stresses $\tau_{x}$ and $\tau_{y}$ increase with an increase the suction parameter.

\section{Conclusion}

An unsteady flow of a viscous incompressible fluid due to non-coaxial rotations of a porous disk and a fluid at infinity subjected to a periodic suction through porous medium has been considered. It is observed that the primary velocity decreases but the secondary velocity increases with an increase in the permeability parameter. It is seen that suction creates thinning of the boundary layer. It is observed that primary velocity decreases with an increase in $\sigma t$ and a reverse effect is observed for secondary velocity. 


\section{Nomenclature}

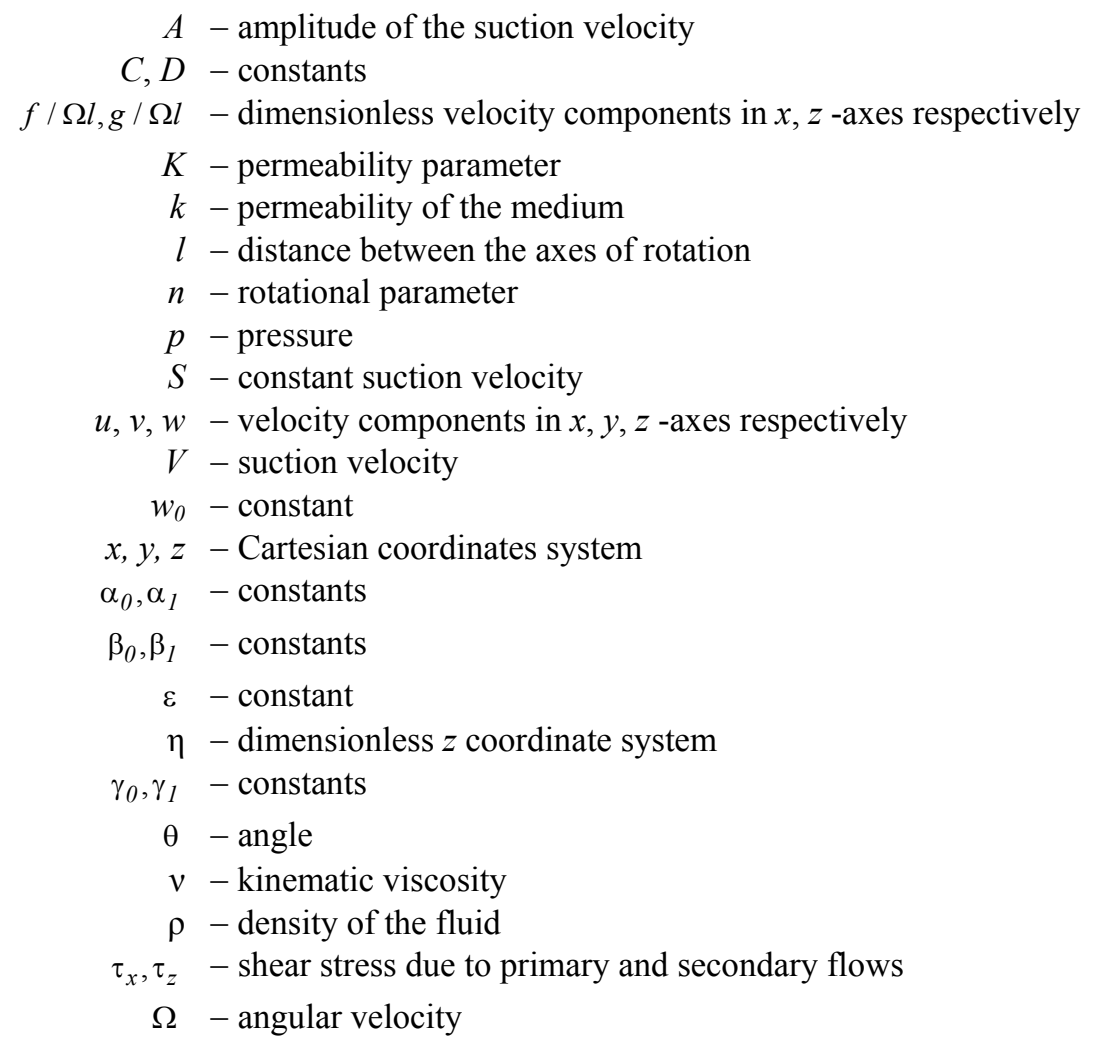

\section{References}

[1] Berker R. (1963): Handbook of Fluid Dynamics. - Berlin: Springer.

[2] Coirier J. (1972): Rotations non-coaxiales d'un disque et d'un fluid à infininity. - J.Dde, Mechanique, vol.11, pp.317-340.

[3] Erdogan M.E. (1976): Non-Newtonian flow due to non-coaxial rotations of a porous disk and a fluid at infinity. Z. Angew. Math. Mech., vol.56, pp.141-146.

[4] Erdogan M.E. (1976): Flow due to eccentric rotating a porous disk and a fluid at infinity. - ASME, J. Appl. Mech., vol.43, pp.203-204.

[5] Erdogan M.E.(1977): Flow due to non-coaxially rotations of a porous disk and a fluid at infinity. - Rev. Roum. Sci Tech Mec, Appl., vol.22, pp.171-178.

[6] Kasiviswanathan S.R. and Rao A.R. (1987): An unsteady flow due to eccentrically rotating porous disk and a fluid at infinity. - Int. J. Engng Sci., vol.25, pp.1419-1425.

[7] Hayat T., Asghar S. and Siddiqui A.M.(1999): Unsteady flow of an oscillating porous disk and a fluid at infinity. - Mecanica, vol.34, pp.259-265.

[8] Guria M., Das B.K. and Jana R.N.(2007): Oscillatory flow due to eccentrically porous disk and a fluid at infinity. - Mecanica, vol.42, pp.487-493.

[9] Guria M., Das S. and Jana R.N. (2007): Hall effects on unsteady flow of a viscous fluid due to non-coaxial rotation of a porous disk and a fluid at infinity. - Int. J. Non-Linear Mech., vol.42, pp.1204-1209.

[10] Ghosh A.K., Paul S. and Debnath Loknath (2002): Unsteady flow induced by variable suction on a porous disk rotating eccentrically with a fluid at infinity. - IJMMS, vol.30, No.4, pp.193-201. 
[11] Guria M., Manna G., Jana R.N. and Pop I. (2007): Flow due to non-coaxial rotations of a permeable disk and a fluid at infinity through a porous medium. - Int. J. Appl. Mech. Eng., vol.12, No.4, pp.965-974.

[12] Hayat T., Ellahi R. and Asghar S. (2007):Unsteady magnetohydrodynamic non-Newtonian flow due to noncoaxial rotations of a disk and a fluid at infinity. - Chem. Eng. Commun., vol.194, pp.37-49.

[13] Hayat T., Ellahi R. and Asghar S. (2008). Hall effects on unsteady flow due to non-coaxially rotating disk and a fluid at infinity. - Chem. Eng. Commun., vol.195, pp.958-976.

[14] Ghara N., Guria M. and Jana R.N. (2012): Hall effects on oscillating flow due to eccentrically rotating porous disk and a fluid at infinity. - Mecanica, vol.47, pp.557-571.

Received: December 16, 2016

Revised: January 5, 2018 